\title{
ENTRE DESAFIOS E OPORTUNIDADES: REFLEXÕES ACERCA DA INSERÇÃO DA DIMENSÃO AMBIENTAL NO CURRÍCULO DE ENGENHARIA DE PRODUÇÃ̃O
}

\section{BETWEEN CHALLENGES AND OPPORTUNITIES: REFLECTIONS CONCERNING THE INCLUSION OF ENVIRONMENTAL DIMENSION IN THE CURRICULUM OF A PRODUCTION ENGINEERING UNDERGRADUATE COURSE}

\author{
Charbel José Chiappetta Jabbour ${ }^{1}$; Fernando César Almada Santos ${ }^{2}$ \\ ${ }^{1}$ Escola de Engenharia de São Carlos da USP - EESC/USP - São Carlos/SP - Brasil \\ cjabbour@terra.com.br \\ ${ }^{2}$ Escola de Engenharia de São Carlos da USP - EESC/USP - São Carlos/SP - Brasil \\ almada@prod.eesc.usp.br
}

\begin{abstract}
Resumo
O objetivo deste artigo é analisar as possibilidades e limitações concernentes à inclusão de princípios da Educação Ambiental (EA) no currículo de um curso de Engenharia de Produção (EP). Segundo a Política Nacional de Educação Ambiental (Lei n. 9.795/99) e as normas internacionais para este tema, a EA deve ser introduzida na totalidade dos conteúdos programáticos já consolidados, isto é, em todas as disciplinas de graduação, à luz de uma perspectiva multi, inter e transdisciplinar. Tendo em vista o pouco conhecimento sistematizado acerca desse tema, realiza-se um resgate histórico do conceito de EA e sua consolidação no Brasil e no mundo. Pautando-se em um estudo de caso, são analisadas as possibilidades e desafios da interiorização dos princípios de EA nas seguintes disciplinas: (a) desenvolvimento de produto; (b) logística; (c) gestão da qualidade; (d) gestão de recursos humanos; e (e) fundamentos de economia. Espera-se, com tais reflexões, contribuir com o aprofundamento do debate sobre EA no âmbito da engenharia de produção, onde ele se mostra ao mesmo tempo fundamental e escasso.
\end{abstract}

Palavras-chave: educação ambiental (EA), currículo, engenharia de produção (EP).

\section{Introdução}

Nas últimas três décadas do século XX, a degradação ambiental se intensificou, como conseqüência da explosão demográfica e acentuação da produção e consumo, inerentes à própria lógica do sistema capitalista. A busca por um Desenvolvimento Sustentável (DS), definido como aquele que atende às necessidades da sociedade atual sem limitar a capacidade das futuras gerações

\footnotetext{
* Esta pesquisa foi realizada no âmbito de um projeto mais amplo apoiado pela Fundação de Amparo à Pesquisa de São Paulo (FAPESP), por meio dos processos de números 06/53549-6 e 05/52741-8.
} 
em atenderem as suas, tornou-se um imperativo (WILKINSON, HILL e GOLLAN, 2001). No cerne do conceito de DS e de sua viabilidade, gravita a EA, que tem por objetivo preparar os seres humanos para o desenvolvimento de uma compreensão integrada do meio ambiente em suas múltiplas e complexas relações. As propostas de EA foram consolidadas no decorrer de diversos eventos internacionais e incorporadas à legislação brasileira pelas Leis n. 6.938/81 e n. 9.795/99. A legislação brasileira coloca a EA como um dever do Estado e das Instituições de Ensino, em todos os níveis de formação, por meio de uma abordagem multi, inter e transdisciplinar, isto é, a EA deve ser introduzida em todas as disciplinas já existentes, podendo-se criar uma específica somente em cursos de pós-graduação (BARBIERI, 2004a).

Assim, o presente artigo analisa a situação atual e discute as principais transformações que podem ser empreendidas em um conjunto de disciplinas que compõem o currículo do curso de graduação em Engenharia de Produção da Escola de Engenharia de São Carlos da Universidade de São Paulo (EESC/USP).

Atualmente, esse curso aborda a EA por meio de uma perspectiva centralizada, limitada a apenas uma disciplina sobre meio ambiente (SHS0176 - Ciências do Ambiente para Engenharia Mecânica e Produção) (USP, 2005), fato que se distancia dos princípios de EA nacionais e internacionais (BARBIERI, 2004b). Dessa forma, este artigo pretende lançar luzes sobre seguintes inquietações:

- As disciplinas da graduação em Engenharia de Produção (EP) da EESC/USP possuem objetivos, programas, métodos e bibliografia que fomentem a Educação Ambiental (EA)?;

- Como essas disciplinas podem ser transformadas para se alinhar aos pressupostos legalistas da EA?; e

- Quais os principais desafios para a futura inserção multi, inter e transdisciplinar da EA no currículo do referido curso?

Para refletir sobre essas indagações, é apresentada a evolução da EA tanto no âmbito internacional quanto nacional, ressaltando a importância dela para os cursos de EP. Logo em seguida são apresentados a metodologia de pesquisa, um breve histórico do curso de EP da EESC/USP, a situação atual da EA nesse curso, bem como potencialidades e desafios.

\section{A Evolução da Educação Ambiental (EA)}

Apesar de os princípios de Educação Ambiental (EA) remontarem às obras de autores clássicos, como Goethe, Rousseau, Humboldt, pode-se considerar o final da Segunda Grande Guerra como o marco histórico da gênese e expansão dos modernos pressupostos da EA, época em 
que o termo passou a ser utilizado. Esses princípios sobre a teoria e a prática da moderna EA desenvolveram-se sob a égide da UNESCO, órgão responsável pela coordenação da maioria das conferências mundiais empreendidas para o debate da causa ambiental. Dessa forma, a moderna EA se desenvolve dentro de uma perspectiva global, que decorre da internacionalização dos desafios ecológicos (BARBIERI, 2004a).

\subsection{O Movimento Internacional da EA}

Vários eventos e marcos institucionais podem ser considerados no plano internacional quando se trata da edificação do conceito de EA, dentre os quais se destacam:

- Conferência das Nações Unidas para o Meio Ambiente Humano (1972), que recomendou a adoção da EA de forma interdisciplinar, a fim de fomentar uma harmonia entre o ser humano e o meio ambiente que o circunda;

- A Carta de Belgrado, Ex-Iugoslávia, elaborada no decorrer do Seminário Internacional sobre EA (1975), que contribuiu para a difusão de objetivos globais da EA em diversos planos (local, regional e nacional), e que devem ser incorporados nos modelos formal e informal de ensinos vigentes;

- Conferência Intergovernamental sobre EA, realizada em Tbilisi, Geórgia, em 1977, que recomendou propostas de EA específicas para a formação de profissionais cujas atividades implicam tomada de decisão sobre a dimensão ecológica, como, por exemplo, administradores, engenheiros, economistas, etc. Como método de ensino, indicou-se o enfoque multidisciplinar, pautado na aprendizagem baseada em problemas e na articulação discente em equipes;

- Conferência Internacional sobre Educação e Formação Ambiental, realizada em Moscou, em 1987, cuja pauta de discussão resultou em fortalecimento das diretrizes propugnadas em Tbilisi;

- Conferência Internacional sobre Meio Ambiente e Sociedade (1997), realizada em Thessaloníki, Grécia, cujas propostas foram ao encontro daquelas discutidas em Tbilisi e em Moscou; e

- A Agenda 21, documento da Conferência das Nações Unidas sobre o Meio Ambiente e Desenvolvimento (1992), que apontou a EA como um catalisador, ou, antes disso, viabilizadora dos postulados inerentes ao conceito de DS. Assim, a EA deve ser vista como um elemento crítico para o sucesso do DS, necessária para que a humanidade equacione a difícil relação entre desenvolvimento econômico e meio ambiente. 
Por se tratarem de proposições internacionais, torna-se fundamental analisar-se como a EA foi institucionalizada no Brasil.

\subsection{A Realidade Brasileira}

No Brasil, a questão ambiental passou a ser discutida com maior amplitude após a democratização da década de 1980, uma vez que os governos militares conceberam a dimensão ecológica como um entrave ao desenvolvimento econômico, sendo a poluição considerada sinônimo de progresso. Por isso, pode-se considerar a EA na legislação ambiental como embrionária até 1981, quando a Lei n.6.938 instituiu a Política Nacional de Meio Ambiente, a qual tornou a EA um dever estatal, que deve garantir a interiorização da dimensão ambiental em todos os níveis do ensino. Na esteira desse processo, a Carta de 1988 incorporou a EA e o DS no texto constitucional. No que tange a Lei de Diretrizes e Bases, que norteia a elaboração de currículos escolares, a EA não é referenciada; muito menos é contemplada como instrumento do DS (BARBIERI, 2004a).

Em 1999, com a promulgação da Política Nacional de Educação Ambiental (BRASIL, 1999), Lei n. 9.795, a educação ambiental passou a ser vista como um componente essencial do sistema nacional de educação, que deve se fazer presente, de forma articulada, em todos os níveis e modalidades do processo educativo, em caráter formal e não-formal. Dessa forma, torna-se imperativo que as instituições de ensino promovam a EA de forma integrada aos programas educacionais vigentes (ABDUL-WAHAB, ABDULRAHEEM e HUTCHINSON, 2003).

\subsubsection{Princípios da EA}

A Lei n. 9.795/99, em seu artigo quarto, declara que os princípios da EA são (BRASIL, 1999):

- Enfoque humanista, holístico, democrático e participativo;

- A concepção do meio ambiente em sua totalidade, considerando a interdependência entre o meio natural, o sócio-econômico e o cultural, sob o enfoque da sustentabilidade;

- O pluralismo de idéias e concepções pedagógicas, na perspectiva da inter, multi e transdisciplinaridade;

- A vinculação entre a ética, a educação, o trabalho e as práticas sociais;

- A garantia de continuidade e permanência do processo educativo;

- A permanente avaliação crítica do processo educativo;

- A abordagem articulada das questões ambientais locais, regionais, nacionais e globais; e

- O reconhecimento e o respeito à pluralidade e à diversidade individual e cultural. 
Como se percebe, os princípios da EA propostos pela legislação brasileira extrapolam a visão meramente conservacionista, pois abordam os pressupostos do DS como um todo. A EA não deve ser tratada apenas pela criação de uma disciplina isolada nos currículos de EP. A dimensão ecológica deve ser tratada transversalmente, e inserida na totalidade dos conhecimentos já consolidados (BARBIERI, 2004a).

\section{A EA em Cursos de Graduação em EP}

Segundo as Diretrizes Curriculares Nacionais do Curso de Graduação em Engenharia, resolução CNE/CES 11/2002, o curso de graduação em Engenharia de Produção objetiva a formação de um profissional generalista, humanista, crítico e reflexivo, capaz de absorver e desenvolver novas tecnologias, estimulando a atuação crítica e criativa na identificação e resolução de problemas, considerando aspectos políticos, econômicos, sociais, ambientais e culturais, com visão ética e humanística, em atendimento às demandas da sociedade (BRASIL, 2002).

A resolução CNE/CES 11/2002, em seu artigo quarto, inciso XI, coloca que um dos objetivos inerentes à adequada formação do engenheiro de produção é desenvolver nele a competência de avaliar o impacto das atividades da engenharia no contexto social e ambiental (BRASIL, 2002).

Essa proposta está de acordo com o movimento mundial de introdução de questões ambientais no âmbito universitário, principalmente no que concerne às atividades de ensino, pesquisa e extensão. Particularmente aos cursos de graduação em engenharia de produção, a inserção da EA no conteúdo programático já consolidado objetiva mostrar aos estudantes como analisar, identificar as causas, efeitos e possíveis soluções que melhorem a qualidade ambiental (ABDUL-WAHAB, ABDULRAHEEM e HUTCHINSON, 2003).

A inserção da EA no currículo de engenharia é fundamental, uma vez que cresce a necessidade dos estudantes se tornarem cidadãos e profissionais que constantemente ponderam a dimensão ecológica no processo de tomada de decisões (CAPDEVILA, BRUNO e JOFRE, 2002), uma vez que a maioria delas produz efeitos para o meio ambiente (MARTIN e HÜTTENHAIN, 2003).

A consolidação da EA nos cursos de EP torna-se ainda mais importante frente a assertiva que os problemas ambientais vigentes são agravados não por falta de conhecimentos tecnológicos, mas sim pela carência de uma visão holística sobre o meio ambiente, que fomente o engajamento dos estudantes de EP em problemáticas ambientais (MARTIN e HÜTTENHAIN, 2003). Entretanto, a maioria dos cursos de engenharia não aborda a dimensão ecológica conforme indicam as principais conferências dedicadas ao tema (MUTMANSKY, 2002). 


\section{Metodologia}

A pesquisa, qualitativa no método, delineada conforme os pressupostos do estudo de caso, objetiva, por meio de dados secundários, a análise da grade curricular do curso de graduação em Engenharia de Produção da Escola de Engenharia de São Carlos da Universidade de São Paulo, Brasil, que foi escolhido por se tratar de um dos melhores cursos de EP do Brasil. A análise do potencial de inserção da dimensão ecológica nas disciplinas desse curso seguiu as seguintes fases: (a) escolha das disciplinas presentes no currículo de EP que apresentam grande importância na formação profissional do engenheiro e para a consecução de programas ambientais, como o de Gestão Ambiental; (b) análise dos objetivos de ensino declarados pelas disciplinas; (c) análise do programa de cada disciplina; (d) análise do método de ensino adotado; (e) análise da bibliografia recomendada. Cada uma dessas perspectivas analíticas foi contraposta com os princípios de EA declarados pela Lei n. 9.795/99. Contudo, antes de se analisar a inserção da EA no curso de EP da EESC/USP, é necessário destacar a história deste curso de graduação.

\section{Resultados}

\subsection{Breve Histórico do Curso de EP da EESC/USP}

O curso de Engenharia de Produção da EESC/USP foi criado em 1969, sendo que em 1975 colou grau a primeira turma de engenheiros de produção formados por essa escola. Anualmente, via vestibular, ingressam 30 discentes. Ressalta-se que esse curso é considerado um dos melhores do Brasil, possui 20 docentes em regime de dedicação integral à docência e à pesquisa, taxa de evasão praticamente nula e empregabilidade de quase todos os formandos.

\subsection{A EA no Curso de Graduação em EP da EESC/USP: situação atual}

As disciplinas escolhidas para a análise da inserção da EA são (USP, 2005):

- SEP0151 - Processo de Desenvolvimento de Produto. A escolha dessa disciplina deve-se ao fato de que alguns especialistas consideram a transformação ecológica nos produtos como uma das mais relevantes para o DS;

- SEP0502 - Administração de Recursos Humanos aplicada à Engenharia de Produção. Essa disciplina foi escolhida por ser relevante para a condução de programas de gestão ambiental empresarial;

- SEP0303 - Logística Integrada, que será analisada por que as atividades logísticas envolvem mecanismos empresariais que vão desde a compra de matéria-prima até a 
distribuição dos produtos acabados aos clientes, inclusive o retorno dos resíduos via fluxos reversos;

- SEP0355 e SEP0356 - Gestão da Qualidade I e II, respectivamente. Essas disciplinas são extremamente importantes para a análise da inserção da EA, pois a gestão da qualidade possui inúmeros paralelismos com a gestão ambiental; e

- SEP0566 - Fundamentos de Economia. A análise da internalização da dimensão ecológica nessa disciplina fornece um contraponto à visão econômica neoclássica, que vê o meio ambiente como um recurso ilimitado, e as ações empresariais de gestão ambiental como entrave à maximização dos lucros.

A Tabela 1 apresenta uma análise do conteúdo programático (objetivos, programa, método e bibliografia) dessas disciplinas à luz dos princípios da EA. Assim, pode-se classificar a EA como fraca, moderada ou forte. A EA fraca é marcada por nenhuma relação entre os pontos de análise e os pressupostos da Lei n. 9.795/99. A EA moderada é aquela em que os aspectos de análise contribuem com os princípios da EA, mas de forma indireta, isto é, não explícita. Por fim, a EA forte se consolida quando objetivos, programa, método ou bibliografia fomentam explicitamente a EA.

Tabela 1 - Análise das disciplinas do currículo de EP da EESC/USP à luz dos princípios de EA

\begin{tabular}{lllll}
\hline Disciplinas & \multicolumn{3}{c}{ Inserção da EA no Conteúdo programático } \\
\cline { 2 - 5 } & Objetivos & Programa & Método & Bibliografia \\
\hline SEP0151 & Ausente & Ausente & Moderado & Ausente \\
\hline SEP0205 & Ausente & Ausente & Moderado & Ausente \\
\hline SEP0303 & Ausente & Ausente & Moderado & Ausente \\
\hline SEP0355 & Ausente & Ausente & Moderado & Ausente \\
\hline SEP0356 & Ausente & Ausente & Moderado & Ausente \\
\hline SEP0566 & Ausente & Ausente & Ausente & Moderado \\
\hline
\end{tabular}

A análise dos objetivos, programa e bibliografia da disciplina SEP0151 evidencia que o alinhamento dessa matéria aos pressupostos e objetivos da EA inexistem. Ressalta-se que, mesmo indiretamente, o método de ensino favorece o trabalho em equipes e resolução de problemas por parte dos alunos, fato que está de acordo com os princípios da EA. Os objetivos da disciplina SEP0502 não relacionam a gestão de recursos humanos e a dimensão ecológica. O programa desta disciplina não orienta as atividades típicas de recursos humanos para a questão ambiental. Implicitamente, o método de aula está de acordo com os princípios da EA, pois fomenta o trabalho em equipe e o desenvolvimento de atividades de resolução de problemas, que potencializam a análise crítica do estudante, auxiliando no desenvolvimento de modelos mentais holísticos e transversais. A bibliografia não contempla temas pertinentes à EA. 
A disciplina SEP0303 apresenta objetivos, programa e bibliografia pouco alinhados aos pressupostos da EA segundo a Lei n. 9.795/99. Apenas o método de ensino se alinha a esses pressupostos, por incentivar atividades de resolução de problemas por meio da articulação de equipes de estudantes. As disciplinas Gestão da Qualidade I (SEP0355) e Gestão da Qualidade II (SEP0356) apresentam essas mesmas características.

Por fim, pode-se dizer que a disciplina SEP0566, Fundamentos de Economia, possui objetivos e programa que não mencionam os princípios da EA em seu texto. O método, baseado essencialmente em aulas teóricas parecem não contribuir para desenvolver modelos metais mais amplos, por meio dos quais os estudantes de engenharia de produção possam analisar o binômio desenvolvimento econômico-meio ambiente. A bibliografia recomendada contempla, de forma moderada, problemáticas ambientais no sumário dos livros recomendados.

\subsection{Potencialidades de Inserção da EA no Currículo de EP da EESC/USP}

Pode-se dizer que há um potencial de integração de princípios da EA no âmbito dessas disciplinas do curso de EP da EESC/USP. No que tange o conteúdo programático da disciplina SEP0151, poderiam ser discutidos: (a) Design For Environment; (b) Análise do Ciclo de Vida do produto; (c) Design para a Desmontagem; e (d) materiais proibidos na composição de produtos.

Já na disciplina SEP0502, poderiam ser abordadas: (a) as contribuições da função de recursos humanos para a gestão ambiental na empresa; (b) o alinhamento das funções típicas de recursos humanos, como seleção, treinamento e benefícios com ênfase em critérios ambientais; (c) a atuação de recursos humanos para o adequado funcionamento do sistema de gestão ambiental na empresa.

O programa da disciplina SEP0303 poderia ressaltar a dimensão ecológica por meio de uma análise de viabilidade ambiental: (a) dos tipos de transporte disponíveis, inclusive combustíveis; (b) das diversas formas de armazenagem de matéria-prima e produtos acabados; (c) da aquisição de matéria prima de fornecedores ambientalmente responsáveis; (d) da gestão de fluxos reversos de materiais, isto é, da logística reversa, que objetiva retornar à firma os componentes do produto que sejam agressivos ao meio ambiente.

Para as disciplinas SEP0355 e SEP0356, sugere-se que incorporem a temática da Gestão da Qualidade Ambiental, cuja filosofia de gestão e instrumentos são similares aos propostos para a Gestão da Qualidade. Ressalta-se que o ideal, segundo a Lei n. 9.795, é que ambas as disciplinas incorporem essas questões, evitando-se a destinação de apenas uma delas para cumprir os princípios da EA. 
A disciplina SEP0566 demonstra um grande potencial de inserção da EA por meio: (a) da demonstração da visão ambiental limitada dos teóricos neoclássicos; (b) da discussão da hipótese de Porter, que afirma que investimentos empresariais em meio ambiente geram vantagens competitivas e inovações para as firmas; (c) da demonstração das implicações das externalidades ambientais negativas da ação das empresas; (d) da dimensão ecológica como nova variável do comércio mundial.

Tabela 2 - Recomendações de conteúdos para a inserção da EA nas disciplinas analisadas

\begin{tabular}{lc}
\hline Disciplinas & Transformações Sugeridas \\
\hline SEP0151 & $\begin{array}{c}\text { Ressaltar como o desenvolvimento de produtos poderá incorporar a dimensão ecológica, desde a geração } \\
\text { da idéia até o lançamento do produto no mercado, por meio da análise do ciclo de vida (REBITZER e } \\
\text { BUXMANN, 2005) }\end{array}$ \\
\hline SEP0502 & $\begin{array}{c}\text { Ressaltar como as atividades típicas da gestão de pessoas podem apoiar a estratégia de gestão ambiental } \\
\text { empresarial e o sistema de gestão ambiental existentes (BOUDREAU e RAMSTAD, 2005; DAILY e } \\
\text { HUANG, 2001) }\end{array}$ \\
\hline SEP0303 & $\begin{array}{c}\text { Identificar as principais decisões logísticas e seus impactos sobre o meio ambiente natural, principalmente } \\
\text { em atividades de transporte, armazenagem, distribuição e gestão dos fluxos reversos (WU e DUNN, }\end{array}$ \\
\hline SEP0355 & $\begin{array}{c}\text { Ressaltar a integração entre a estratégia de gestão da qualidade e a gestão ambiental, principalmente por } \\
\text { meio dos paralelismos existentes entre as normas das séries ISO 9000 e ISO 14000 (LAWRENCE, } \\
\text { ANDREWS e FRANCE, 1998) }\end{array}$ \\
\hline SEP0566 & $\begin{array}{l}\text { Inserir conteúdos de economia do meio ambiente, valoração de recursos naturais, ressaltando a visão } \\
\text { limitada dos pressupostos neoclássicos da teoria econômica. Evidenciar que o investimento da firma } \\
\text { em questões ambientais pode contribuir para a geração de inovaçoses em produtos e processos } \\
\text { (BARBIERI, 2004b). }\end{array}$ \\
\hline
\end{tabular}

Além dessas alterações no programa da disciplina, recomenda-se que:

- Os objetivos das disciplinas explicitem o alinhamento da matéria com os princípios da EA;

- Os métodos didáticos continuem a apoiar a abordagem de problemas por meio de equipes multidisciplinares, explicitando a importância do método para a EA; e

- A bibliografia contenha material relacionado à dimensão ambiental.

Apesar dessas potencialidades, a inserção da EA no currículo de EP da EESC/USP apresenta diversos desafios, dos quais destacam-se:

- A necessidade de os professores também serem preparados para lidar com a EA;

- A necessidade de constante comunicação e sincronia entre as atividades dos docentes em matéria de EA, para fortalecer o caráter multi, inter e transdisciplinar da EA;

- A burocratização dos mecanismos para alteração curricular da USP;

- A visão dos docentes de que a integração pontual da questão ambiental no currículo, por meio da criação de uma disciplina isolada, é uma medida satisfatória; e

- O número restrito de docentes cujas linhas de pesquisa são voltadas para a EA. 


\title{
6. Considerações Finais
}

A intensificação da degradação ambiental tornou necessário um novo padrão de desenvolvimento, mais sustentável, apoiado por um sistema educacional em que esteja presente a dimensão ecológica, segundo uma série de princípios. Este artigo mostrou que os princípios da EA, referendados tanto nos fóruns internacionais quanto pelas instituições brasileiras não estão sendo considerados no currículo do curso de graduação em Engenharia de Produção da EESC/USP. A análise de algumas matérias permite a conclusão de que os objetivos, programas, métodos e bibliografia indicam indiferença em relação aos pressupostos e objetivos da EA. Esse curso apresenta uma integração pontual da EA em seu currículo, por meio de criação de disciplina específica para a abordagem da dimensão ecológica, o que é confirmado pela literatura como uma tendência pouco eficaz. Entretanto, a inserção da EA no currículo de graduação em EP é obstaculizada por diversos problemas.

Ressalta-se que, pelo caráter restrito da pesquisa, os resultados desse estudo de caso não podem ser extrapolados, nem para o entendimento da realidade brasileira, nem como demonstrativo do movimento internacional. Por isso, recomenda-se que estudos mais abrangentes e profundos sejam conduzidos no Brasil e em outros países, por meio de construções comparadas e multicomparadas, que permitam a identificação de melhores práticas.

\begin{abstract}
The aim of this article is to analyze the possibilities and limitations of the inclusion of Environmental Education (EE) in an undergraduate course in the field of Production Engineering (PE). According to the National Policy of Environmental Education (Law n.9.795/9) and the international norms linked to this thematic, EE has to be introduced in the whole set of a consolidated knowledge in the light of a perspective which involves, mixes, and makes the subjects of an undergraduate course interact. Considering the scarce systematized knowledge of this thematic, a historical analysis of the concept of EE and its consolidation in Brazil and in the world is carried out. Based on a case study, the inclusion of EE in the following subjects of the PE is analyzed: (a) product development; (b) logistics; (c) quality management; (d) human resource management; and (e) fundamentals of economics. These reflections are expected to contribute to intensify the debate about EE in the field of Production Engineering where it is concurrently essential and scarce.
\end{abstract}

Keywords: environmental education (EE), syllabus, production engineering (PE). 


\section{Referências}

ABDUL-WAHAB, S.A.; ABDULRAHEEM, M.Y. \& HUTCHINSON, M. The need for inclusion of environmental education in undergraduate engineering curricula. International Journal of Sustainability in Higher Education. Vol. 4, n. 2, p.126-137, 2003.

cross $r$ ref

BARBIERI, J.C. Educação ambiental e a gestão ambiental em cursos de graduação em administração: objetivos, desafios e propostas. Revista de Administração Pública. Rio de Janeiro, Vol.38, n.6, p.919-946, 2004.

BARBIERI, J.C. Gestão ambiental empresarial, São Paulo, Saraiva, 2004.

BOUDREAU, J.W. \& RAMSTAD, P.M. Talentship, talent segmentation and sustainability: a new HR decision science paradigm for a new strategy definition. Human Resource Management. Vol.44, n.2, p.129-136, 2005.

cross $^{\text {ref }}$

BRASIL. "Lei No. 9.795 de 27 de abril de 1999. Institui a Política Nacional de Educação Ambiental”, Brasília, DF, Diário Oficial da União, 28 de abril de 1999.

BRASIL. "Resolução CNE/CES 11, de 11 de março de 2002. Institui Diretrizes Curriculares Nacionais do Curso de Graduação em Engenharia”, Brasília, MEC, 11 de março de 2002.

CAPDEVILA, I.; BRUNO, J. \& JOFRE, L. Curriculum greening and environmental research co-ordination at the Technical University of Catalonia, Barcelona. Journal of Cleaner Production. Vol.10, n.1, p.25-31, 2002.

cross ${ }^{\text {ref }}$

DAILY, B.F. \& HUANG, S. Achieving sustainability through attention to human resource factors in environmental management. International Journal of Operations \& Production Management. Vol.21, n.12, p.1539-1552, 2001.

cross ${ }^{\text {ref }}$

LAWRENCE, L.; ANDREWS, D. \& FRANCE, C. Alignment and deployment of environmental strategy through total quality management. The TQM Magazine. Vol.10, n.4, p.238-245, 1998.

cross ${ }^{\text {ref }}$

MARTIN, F.G. \& HÜTTENHAIN, S.H. Environmental education: new paradigms and engineering syllabus. Journal of Cleaner Production. Vol.11, n.3, p.247-251, 2003.

cross ${ }^{\text {ref }}$

MUTMANSKY, J.M. Environmental education in mining engineering curricula: perceptions of practicing engineers.

Mining Engineering. Vol.54, n.4, p.35-39, 2002.

REBITZER, G. \& BUXMANN, K. The role and implementation of LCA within life cycle management at Alcan. Journal of Cleaner Production. Vol.13, n.14, p.1327-1335, 2005.

cross ${ }^{\text {ref }}$

SEIFFERT, M.E.B. \& LOCH, C. Systemic thinking in environmental management: support for sustainable development. Journal of Cleaner Production. Vol.13, n.12, p.1197-1202, 2005.

cross ${ }^{\text {ref }}$

Universidade de São Paulo - USP. “Grade curricular do curso de Engenharia de Produção da EESC", Acesso em: 03/10/2005, Disponível em <www.sistemas.usp.br>, 2005.

WILKINSON, A.; HILL, M. \& GOLLAN, P. The sustainability debate. International Journal of Operations \& Production Management. Vol. 21, n.12, p.1492-1502, 2001.

cross ${ }^{\text {ref }}$

WU, H. \& DUNN, S.C. Environmentally responsible logistics systems. International Journal of Physical Distribution \& Logistics Management. Vol.25, n.2, p.20-37, 1995.

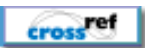




\section{Dados dos autores:}

Charbel José Chiappetta Jabbour

Escola de Engenharia de São Carlos da USP

Departamento de Engenharia de Produção

Doutorando em Engenharia de Produção pela EESC/USP

Av. Dr. Carlos Botelho, 2791, Centro, São Carlos/SP, Brasil, CEP: 13560-251.

Telefone: $163373-8286$

e-mail:cjabbour@terra.com.br

Fernando César Almada Santos

Escola de Engenharia de São Carlos da USP

Departamento de Engenharia de Produção

Professor Doutor

Av. Trabalhador São-carlense, 400, Centro, São Carlos/SP, Brasil, CEP: 13566-590.

Telefone: 16 3373-3985

e-mail:almada@prod.eesc.usp.br

Recebido para publicação em: 21/10/2006

Aceito para publicação em: 03/12/2006 